\title{
Stemming bone loss by suppressing apoptosis
}

\author{
Janet M. Hock
}

Commentary

See related article

this issue, pages 439-446.

Lilly Research Labs, Indianapolis, Indiana 46285, USA. Phone: (317) 276-6074;

Fax: (317) 578-8803; E-mail: hock_janet_m@lilly.com.

It's been over 100 years since the discovery of parathyroid hormone (PTH), yet the in vivo mechanisms of action of PTH at the cellular and molecular levels are still not well understood. Despite a wealth of in vitro studies, none predicted the anabolic effect of PTH on the skeleton. The anabolic effect of PTH was initially observed using parathyroid gland extract (PTE) in young rats and rabbits $(1,2)$. At the time, the models were thought to reproduce, in part, the pathologic processes of hyperparathyroidism, in which an early destructive phase was followed by an ebullient reparative phase of bone formation (1). After the successful synthesis of hPTH 1-34 in the early 1970s (3), its anabolic phenomenon was revisited, and small clinical trials suggested that PTH could be used as an anabolic therapy for osteoporosis $(4,5)$. The data were viewed with scepticism, because the literature at the time was dominated by the notion that PTH was a hormone controlling calcium homeostasis, in part by stimulating bone resorption. In vitro, embryonic bone organ culture studies confirmed the original in vivo studies with PTE, by showing that PTH initially stimulated resorption and had a delayed, stimulatory effect on the rate of matrix synthesis (6). These data supported the concept of "coupling" between resorption and formation, with PTH as a key stimulator of bone turnover (6). However, in vivo data from rats and humans showed that PTH stimulated bone formation de novo, without a prior episode of resorption $(7,8)$.

The need to better understand how once-daily injections of PTH increase bone mass in osteoporotic humans has intensified as we have realized the magnitude of the health care challenges and costs in caring for osteoporosis. The most recent clinical studies of the effects of PTH on bone of osteoporotic women (9-11) show increases in bone mass superior to those induced by known anti-resorptives $(4,5)$. Studies of ovariectomized monkeys show that the rapid increases in bone mass induced by PTH at the spine and hip are associated with increases in biomechanical strength (12, 13). Rabbits, one of the smallest animal models with osteonal cortical bone, were used to explore the effects on cortical bone (14). However, it is rodents that are the preferred model for exploring the PTH-induced anabolic mechanisms of action (5). In this issue of the JCI, Jilka et al. (15) report the use of a mouse model to support the hypothesis that increased longevity of osteoblasts, due to inhibition of their apoptosis by PTH, may explain the significant increase in bone forming surfaces that is a prerequisite for new bone matrix accrual.

There is prior evidence that anabolic agents may work through stimulation of cell proliferation and differentiation (16). In vitro, the effects of PTH on proliferation are inconsistent and depend on the bone cell or organ model used (17-20). Some cell lines, such as the osteoblast-like osteosarcoma cells, may lack cell-cycle genes, such as p53 and $\mathrm{Rb}$, or may express mutant forms of these genes. Bone organ culture, or bone cells isolated from fetal or neonatal animals, may not respond to hormonal stimulation under the same constraints as those from postnatal and mature rodents (21). In vivo, there is currently no evidence to support PTH as a stimulator of proliferation. In young rodents, proliferating cells in bone are located subjacent to the growth plate, the cortical endosteum of the metaphysis, and the cortical periosteum of the diaphysis (22-24). These are all locations in which PTH exerts its stimulatory effect on bone formation (25-28). In young rats with abundant proliferating cell populations, PTH appears to select proliferating cells in Sphase, and commits an increasing number to the osteoblast lineage within 24 hours after the first injection $(25,29)$. In mature rats, PTH stimulates lining cells on quiescent surfaces to function as osteoblasts $(30,31)$. The concept of regulation of progenitor cell differentiation by PTH is also supported by comparisons of the effects on bone histology of once-daily PTH injections with continuous infusion. In contrast to the increase in osteoblasts associated with once-daily PTH injections, continuous infusion of PTH favors a fibroblastic pathway in PTH-responsive cells, as marrow fibrosis is observed close to bone surfaces (32), and there is modulation of IGF-binding proteins (33). The mechanisms that lead to increased bone formation or increased fibrosis by different regimens of PTH in rodents are unknown.

In considering the interplay between proliferation, differentiation, and apoptosis, differences between rodent models should be recognized. Unlike the effect in rats, little is known of the anabolic effect of PTH in mice. The effects on calcium homeostasis in mice given PTH have still to be characterized. PTH does not increase bone mass in many mouse strains (M. Sato et al., unpublished data). The SAMP6 and SAMR1 mouse strains are therefore of interest, as PTH increased bone mass. Evidence

\section{Recent clinical studies of the effects of PTH on bone of osteoporotic women show increases in bone mass superior to those induced by known anti-resorptives.}

that PTH regulates osteoblast differentiation and function in vivo is abundant and, up to now, has been widely accepted as the mechanism of action for PTH $(4,5)$. Histomorphometry of bone in humans and a number of animal species, including the mouse study by Jilka et al., shows increased osteoblast numbers and bone forming surface, in the absence of a concomitant increase in resorption. However, the histomorphometric changes and dose of PTH required to demonstrate this anabolic outcome in the SAMP6 and SAMR1 mice suggest that there may be differences in the mechanisms by which bone 
mass is increased in rats and mice. The dose of $400 \mu \mathrm{g} / \mathrm{kg}$ per day is far in excess of that used in rats, in which doses as low as $2 \mu \mathrm{g} / \mathrm{kg}$ per day increase trabecular bone mass (34). Bone histomorphometry of the PTH-treated mice demonstrated a surprising increase in osteoid surface area, which has not been reported in most rat studies.

The studies by Jilka et al. were done after 1 month of treatment, equivalent to exposure of bone to PTH for 4 bone turnover cycles, when the cellular marrow milieu may be significantly different from that in the early stages of treatment. Thus, it is possible that other mechanisms come into play during an extended phase of treatment. Mature osteoblasts and cells within the hematopoietic bone marrow synthesize a wide array of cytokines, and express receptors for many of these factors $(15$, $35)$. The bone growth factors IGF-1, TGF- $\beta$, and bFGF, and their receptors, can be expressed by osteoblasts and may mediate some PTH-induced responses in vitro $(15,36)$. These factors could mediate the late events to represent an alternate mechanism by which PTH retains osteoblasts and stimulates their function over longer periods of time.

The increase in osteoid surfaces may be associated with a delay in apoptosis and increased osteoblast longevity in mice, as the final phase of osteoblast differentiation depends upon maturation of the underlying matrix and its mineralization. In young PTH-treated rats that do not exhibit increased osteoid surfaces (7), PTH rapidly stimulates synthesis of matrix metalloproteinases (MMPs), such as MMP13 (collagenase I) and MMP9 (gelatinase B), as an integral component of its early mechanisms of action $(37,38)$. One hypothesis holds that MMPs are required to recondition unmineralized bone surfaces, resulting in the detachment of mature cells and the recruitment and differentiation of additional osteoprogenitors $(39,40)$. Given that detachment of mature cells from their extracellular matrix is a signal for apoptosis, these events would imply increased apoptosis of mature osteoblasts. Preliminary data suggest that such an event may be an early event in the response to PTH (41).

Based on work in growth and development fields, the accepted concept that osteoblasts cover the bone surface as sheets of cells, and the relatively short life of osteoblasts, apoptosis within this cell population is unlikely to be a random event. Because the rodent skeleton continues to develop throughout most of life, owing to at least 1 active growth plate in most bones, experimentally induced responses in rodents may reflect effects on growth processes and bone morphogenesis. In rats, PTH stimulates bone formation at sites of development of the new metaphyseal bone to modify trabecular 3dimensional geometry by increasing trabecular thickness and connectivity. These effects can be attributed to effects on both growth processes (42) and stimulation of bone modeling in which de novo bone formation occurs without prior resorption on that surface. An effect on apoptosis would provide a cellular mechanism to explain the alterations in trabecular geometry attributed to PTH. The region of interest in which apoptotic cells were evaluated by Jilka et al. is contained within an area of the metaphysis that appears to undergo trabecular remodeling in mice. However, there were differences between what was observed and what has been reported by others for large animal models, where remodeling has been more extensively characterized. In large animals and humans, PTH stimulates significant restructuring of bone by intratrabecular tunneling and intracortical remodeling (effects not observed in rodents), in addition to stimulating apposition of new matrix on endosteal surfaces (43). Although it is possible that increased osteoblast longevity due to inhibition of apoptosis would prolong the bone formation period, there is no evidence that PTH modifies the formation period of a bone turnover cycle (44).

Caution is necessary in interpreting results of a single in vivo model. Apoptosis was a rare event in the study by Jilka et al., affecting less than $2 \%$ of the cells in controls, and identification of apoptotic cells by TUNEL staining often requires a second, apoptosis-oriented method for confirmation. The published literature has consistently shown PTH rapidly induces bone formation by increasing the numbers of functional osteoblasts, consistent with a pronounced effect on osteoblast differentiation $(4,5)$. These observations, together with the limitations of in vitro and rodent models in predicting mechanisms that may be relevant to humans, infer that apoptosis is unlikely to be the exclusive mechanism by which PTH increases bone mass in vivo. However, Jilka et al. have contributed to our understanding that apoptosis may have a role in the varied mechanisms by which PTH regulates in vivo anabolic effects.

1.Jaffe, H. 1933. Hyperparathyroidism (Recklinghausen's disease of bone). Arch. Pathol. 16:63-122.

2. Parsons, J.A. 1976. Parathyroid physiology and the skeleton. In Biochemisty and physiology of bone. G. Bourne, editor. Academic Press. New York, NY. 271-298.

3. Potts, J., et al. 1995. Parathyroid hormone: physiology, chemistry, biosynthesis, secretion, metabolism and mode of action. In Endocrinology. L. DeGroot, editor. W.B. Saunders Co. Philadelphia, PA. 920-966.

4. Cosman, F., and Lindsay, R. 1998. Is parathyroid hormone a therapeutic option for osteoporosis? A review of the clinical evidence. Calcif. Tissue Int. 62:475-480.

5. Dempster, D.W., Cosman, F., Parisien, M., Shen, V., and Lindsay, R. 1993. Anabolic actions of parathyroid hormone on bone. Endocr. Rev. 14:690-709.

6. Howard, G.A., Bottemiller, B.L., and Baylink, D.J. 1980. Evidence for the coupling of bone formation to bone resorption in vitro. Metab. Bone Dis. Relat. Res. 2:131-135.

7. Hock, J.M., Hummert, J.R., Boyce, R., Fonseca, J., and Raisz, L.G. 1989. Resorption is not essential for the stimulation of bone growth by human parathyroid hormone 1-34 in rats in vivo. J. Bone Miner. Res. 4:449-458.

8. Hodsman, A., and Steer, B. 1993. Early histomorphometric changes in response to parathyroid hormone therapy in osteoporosis: evidence for de novo bone formation on quiescent cancellous surfaces. Bone. 14:523-527.

9. Lindsay, R., et al. 1997. Randomised controlled study of effect of parathyroid hormone on vertebral-bone mass and fracture incidence among post-menopausal women on oestrogen with osteoporosis. Lancet. 350:550-555.

10. Hodsman, A.B., et al. 1997. A randomized controlled trial to compare the efficacy of cyclical parathyroid hormone versus cyclical parathyroid hormone and sequential calcitonin to improve bone mass in postmenopausal women with osteoporosis. J. Clin. Endocrinol. Metab. 82:620-628.

11. Lane, N., et al. 1998. Parathyroid hormone treatment can reverse corticosteroid-induced osteoporosis. J. Clin. Invest. 102:1627-1633.

12. Sato, M., et al. 1998. 3D finite element modeling of the effects of recombinant hPTH (1-34) on the lumbar vertebra of Cynomolgus macaca. Orthopedic Research Society Meeting. Osaka, Japan. September 28-30, 1998.

13. Brommage, R., et al. 1999. Daily treatment with recombinant human parathyroid hormone, LY333334, rhPTH 1-34, for one year increases bone mass in ovariectomized monkeys. J. Clin. Endocrinol. Metab. In press.

14. Hirano, T., Burr, D., Turner, C., Sato, M., and Hock, R.C.J. 1999. Anabolic effects of human biosynthetic parathyroid hormone fragment (134 ) on remodeling and mechanical properties of cortical bone in rabbits. J. Bone Miner. Res. 14:536-545.

15. Jilka, R.L., et al. 1999. Increased bone formation by prevention of osteoblast apoptosis with parathyroid hormone. J. Clin. Invest. 104:439-446.

16. Canalis, E., Pash, J., and Varghese, S. 1993. Skeletal growth factors. Crit. Rev. Eukaryot. Gene Expr. 
3:155-166

17. Onishi, T., and Hruska, K. 1997. Expression of p27Kip1 in osteoblast-like cells during differentiation with parathyroid hormone. Endocrinology. 138:1995-2004.

18. Partridge, N., Opie, A., Opie, R., and Martin, T. 1985. Inhibitory effects of parathyroid hormone on growth of osteogenic sarcoma cells. Calcif. Tissue Int. 37:519-525.

19. Verheijen, M., and Defize, L. 1995. Parathyroid hormone inhibits mitogen-activated protein kinase activation in osteosarcoma cells via a protein kinase A-dependent pathway. Endocrinology. 136:3331-3337.

20. Chaudhary, L. and Avioli, L. 1998. Identification and activation of mitogen-activated protein (MAP) kinase in normal human osteoblastic and bone marrow stromal cells: attenuation of MAP kinase activation by cAMP, parathyroid hormone and forskolin. Mol. Cell. Biochem. 178:59-68.

21. Canalis, E., Hock, J.M., and Raisz, L.G. 1994. Anabolic and catabolic effects of parathyroid hormone on bone and interactions with growth factors. In The parathyroids. J.P. Bilezekian and M.A. Levine, editors. Raven Press. New York, NY. 65-82.

22. Kember, N.F. 1960. Cell division in endochondral ossification. A study of cell proliferation in rat bones by the method of tritiated thymidine autoradiography. J. Bone Joint Surg. Br. 42:824-839.

23. Kimmel, D.B., and Jee, W.S.S. 1980. Bone cell kinetics during longitudinal bone growth in the rat. Calcif. Tissue Int. 32:123-133.

24. Owen, M.E. 1970. The origin of bone cells. Int. Rev. Cytol. 28:213-218.

25. Hock, J.M., et al. 1994. Anabolic PTH targets proliferating cells of the primary spongiosa in young rats, and increases the number differentiating into osteoblasts. J. Bone Miner. Res. 9(Suppl.1):5421.

26. Onyia, J., Bidwell, J., Herring, J., Hulman, J., and Hock, J. 1995. In vivo, human parathyroid hor- mone fragment (hPTH 1-34) transiently stimulates immediate early response gene expression, but not proliferation, in trabecular bone cells of young rats. Bone. 17:479-484

27. Young, R.W. 1964. Specialization of bone cells. Symposium on bone biodynamics. H.M. Frost, editor. Little, Brown and Co. Boston, MA. 120-132.

28. Gunness-Hey, M. and Hock, J.M. 1989. Loss of anabolic effect of parathyroid hormone on bone after discontinuation of hormone in rats. Bone. 10:447-452

29. Onyia, J., et al. 1997. Proliferating cells in the primary spongiosa express the osteoblast phenotype in vitro. Bone. 20:93-100.

30. Leaffer, D., et al. 1995. Modulation of osteogenic cell ultrastructure by RS-23581, an analog of human parathyroid hormone (PTH)-related peptide-(1-34) and bovine PTH-(1-34). Endocrinology. 136:3624-3631

31. Dobnig, H., and Turner, R. 1995. Evidence that intermittent treatment with parathyroid hormone increases bone formation in adult rats by activation of bone lining cells. Endocrinology. 136:3632-3638.

32. Turner, R., Evans, G., Cavolina, J., Halloran, B., and Morey-Holton, E. 1998. Programmed administration of parathyroid hormone increases bone formation and reduces bone loss in hindlimbunloaded ovariectomized rats. Endocrinology. 139:4086-4091.

33. Watson, P., et al. 1999. Enhanced osteoblast development after continuous infusion of $\mathrm{hPTH}$ (1-84) in the rat. Bone. 24:89-94.

34. Gunness-Hey, M. and Hock, J.M. 1984. Increased trabecular bone mass in rats treated with synthetic parathyroid hormone. Metab. Bone Dis. Relat. Res. 5:177-181.

35. Watson, P., Watson, A., and Hodsman, A. 1996. Expression of growth factor ligand and receptor genes in rat cancellous bone trabeculae and marrow. J. Mol. Endocrinol. 17:45-54.
36. Pfeilschifter, J., et al. 1993. Mitogenic responsiveness of human bone cells in vitro to hormones and growth factors decreases with age. J. Bone Miner. Res. 8:707-717.

37.Zhao, W., Byrne, M., Boyce, B., and Krane, S. 1999. Bone resorption induced by parathyroid hormone is strikingly diminished in collagenase-resistant mutant mice. J. Clin. Invest. 103:517-524.

38. McClelland, P., et al. 1998. Intermittent administration of parathyroid hormone (1-34) stimulates matrix metalloproteinase-9 (MMP-9) expression in long bone. J. Cell. Biochem. 70:391-401.

39. Jimenez, M., et al. 1999. Collagenase 3 is a target of Cbfa1, a transcript factor of the runt gene family involved in bone formation. Mol. Cell. Biol. 19:4431-4442.

40. Partridge, N., et al. 1996. The regulation and regulatory role of collagenase in bone. Crit. Rev. Eukaryot. Gene Expr. 6:15-27.

41. Yang, X., et al. 1997. Selective stimulation of apoptosis in trabecular osteoblasts and osteocytes of young rats treated with once daily hPTH 1-34 to increase bone mass. J.Bone. Miner. Res. 12(Suppl. 1):S316. (Abstr.)

42. Hock, J.M., and Fonseca, J. 1990. Anabolic effect of human synthetic parathyroid hormone (1-34) depends on growth hormone. Endocrinology. 127:1804-1810.

43. Jerome, C., Gladwell, T., Scheer, K., Brommage, R., and Hock, J. 1998. Increased cancellous bone connectivity and conserved cortical bone mass in PTH (1-34) (LY333334)-treated monkeys. XXVIth European Symposium on Calcified Tissues. Maastricht, the Netherlands.

44. Boyce, R., Paddock, C., Franks, A., Jankowsky, M., and Eriksen, E. 1996. Effects of intermittent hPTH (1-34) alone and in combination with $1,25(\mathrm{OH}) 2 \mathrm{D} 3$ or risedronate on endosteal bone remodeling in canine cancellous and cortical bone. J. Bone Miner. Res. 11:600-613. 